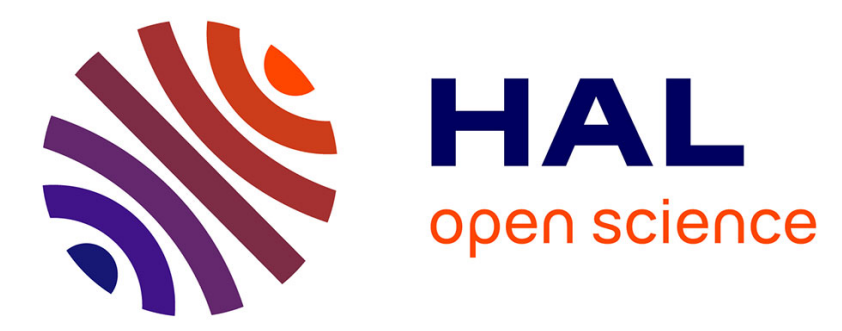

\title{
Measurement of the optical parameters of the Virgo interferometer
}

F. Acernese, P. Amico, M. Alshourbagy, F. Antonucci, S. Aoudia, P. Astone, S. Avino, D. Babusci, G. Ballardin, F. Barone, et al.

\section{- To cite this version:}

F. Acernese, P. Amico, M. Alshourbagy, F. Antonucci, S. Aoudia, et al.. Measurement of the optical parameters of the Virgo interferometer. Applied optics, 2007, 46, pp.3466-3484. in2p3-00113562

\section{HAL Id: in2p3-00113562 \\ https://hal.in2p3.fr/in2p3-00113562}

Submitted on 14 Nov 2006

HAL is a multi-disciplinary open access archive for the deposit and dissemination of scientific research documents, whether they are published or not. The documents may come from teaching and research institutions in France or abroad, or from public or private research centers.
L'archive ouverte pluridisciplinaire $\mathbf{H A L}$, est destinée au dépôt et à la diffusion de documents scientifiques de niveau recherche, publiés ou non, émanant des établissements d'enseignement et de recherche français ou étrangers, des laboratoires publics ou privés. 


\title{
Measurement of the optical parameters of the VIRGO interferometer
}

\author{
Virgo Collaboration
}

F. Acernese ${ }^{6}$, P. Amico ${ }^{10}$, M. Alshourbagy ${ }^{11}$, F. Antonucci ${ }^{12}$, S. Aoudia ${ }^{7}$, P. Astone $^{12}$, S. Avino ${ }^{6}$, D. Babusci ${ }^{4}$, G. Ballardin ${ }^{2}$, F. Barone ${ }^{6}$, L. Barsotti ${ }^{11}$, M. Barsuglia ${ }^{8}$, F. Beauville ${ }^{1}$, S. Bigotta ${ }^{11}$, S. Birindelli ${ }^{11}$, M.A. Bizouard ${ }^{8}$, C. Boccara ${ }^{9}$, F. Bondu ${ }^{7}$, L. Bosi ${ }^{10}$, C. Bradaschia ${ }^{11}$, S. Braccini ${ }^{11}$, A. Brillet ${ }^{7}$, V. Brisson ${ }^{8}$, L. Brocco ${ }^{12}$, D. Buskulic ${ }^{1}$, E. Calloni ${ }^{6}$, E. Campagna ${ }^{3}, \mathrm{~F}$. Carbognani $^{2}$, F. Cavalier ${ }^{8}$, R. Cavalieri ${ }^{2}$, G. Cella ${ }^{11}$, E. Cesarini ${ }^{3}$, E. Chassande-Mottin ${ }^{7}$, N. Christensen ${ }^{2}$, C. Corda ${ }^{11}$, A. Corsi ${ }^{12}$, F. Cottone ${ }^{10}$, A.-C. Clapson ${ }^{8}$, F. Cleva ${ }^{7}$, J.-P. Coulon ${ }^{7}$, E. Cuoco ${ }^{2}$, A. Dari' ${ }^{10}$, V. Dattilo ${ }^{2}$, M. Davier ${ }^{8}$, M. del Prete ${ }^{2}$, R. De Rosa ${ }^{6}$, L. Di Fiore ${ }^{6}$, A. Di Virgilio ${ }^{11}$, B. Dujardin ${ }^{7}$, A. Eleuteri ${ }^{6}$, I. Ferrante ${ }^{11}$, F. Fidecaro ${ }^{11}$, I. Fiori ${ }^{11}$, R. Flaminio ${ }^{1,2}$, J.-D. Fournier ${ }^{7}$, O.Francois ${ }^{2}$, S. Frasca ${ }^{12}$, F. Frasconi ${ }^{2}{ }^{11}$, L. Gammaitoni ${ }^{10}$, F. Garufi ${ }^{6}$, E. Genin ${ }^{2}$, A. Gennai ${ }^{11}$, A. Giazotto ${ }^{11}$, G. Giordano ${ }^{4}$, L. Giordano ${ }^{6}$, R. Gouaty ${ }^{1}$, D. Grosjean ${ }^{1}$, G. Guidi ${ }^{3}$, S. Hebri ${ }^{2}$, H. Heitmann ${ }^{7}$, P. Hello ${ }^{8}$, S. Karkar ${ }^{1}$, S. Kreckelbergh ${ }^{8}$, P. La Penna ${ }^{2}$, M. Laval ${ }^{7}$, N. Leroy ${ }^{8}$, N. Letendre ${ }^{1}$, B. Lopez ${ }^{2}$, Lorenzini ${ }^{3}$, V. Loriette ${ }^{9}$, G. Losurdo ${ }^{3}$, J.-M. Mackowski ${ }^{5}$, E. Majorana ${ }^{12}$, C. N. Man ${ }^{7}$, M. Mantovani ${ }^{11}$, F. Marchesoni ${ }^{10}$, F. Marion ${ }^{1}$, J. Marque ${ }^{2}$, F. Martelli ${ }^{3}$, A. Masserot ${ }^{1}$, M. Mazzoni ${ }^{3}$, L. Milano ${ }^{6}$, F. Menzinger $^{2}$, C. Moins ${ }^{2}$, J. Moreau ${ }^{9}$, N. Morgado ${ }^{5}$, B. Mours ${ }^{1}$, F. Nocera ${ }^{2}$,

A. Pai ${ }^{12}$, C. Palomba ${ }^{12}$, F. Paoletti ${ }^{2}, 11$, S. Pardi ${ }^{6}$, A. Pasqualetti ${ }^{2}$, R. Passaquieti ${ }^{11}$, D. Passuello ${ }^{11}$, B. Perniola ${ }^{3}$, F. Piergiovanni ${ }^{3}$, L. Pinard ${ }^{5}$, R. Poggiani ${ }^{11}$, M. Punturo ${ }^{10}$, P. Puppo ${ }^{12}$, K. Qipiani ${ }^{6}$, P. Rapagnani ${ }^{12}$, V. Reita ${ }^{9}$, A. Remillieux ${ }^{5}$, F. Ricci ${ }^{12}$, I. Ricciardi ${ }^{6}$, P. Ruggi ${ }^{2}$, G. Russo ${ }^{6}$, S. Solimeno ${ }^{6}$, A. Spallicci ${ }^{7}$, R. Stanga ${ }^{3}$, M. Tarallo ${ }^{11}$, M. Tonelli ${ }^{11}$, A. Toncelli ${ }^{11}$, 


\section{E. Tournefier ${ }^{13}$, F. Travasso ${ }^{10}$, C. Tremola ${ }^{11}$, G. Vajente ${ }^{11}$, D. Verkindt ${ }^{1}$,} F. Vetrano ${ }^{3}$, A. Viceré ${ }^{3}$, J.-Y. Vinet ${ }^{7}$, H. Vocca ${ }^{10}$ and M. Yvert ${ }^{1}$

${ }^{1}$ Laboratoire d'Annecy-le-Vieux de physique des particules (LAPP), IN2P3/CNRS, Université de Savoie, BP 110, F-74941, Annecy-le-Vieux, CEDEX, France;

${ }^{2}$ European Gravitational Observatory (EGO), Via E. Amaldi, I-56021 Cascina (PI) Italia;

${ }^{3}$ INFN - Sezione Firenze/Urbino Via G.Sansone 1, I-50019 Sesto Fiorentino; and/or Università di Firenze, Largo E. Fermi 2, I - 50125 Firenze and/or Università di Urbino, Via S.Chiara, 27 I-61029 Urbino, Italia;

${ }^{4}$ INFN, Laboratori Nazionali di Frascati Via E. Fermi, 40, I-00044 Frascati (Roma) Italia;

${ }^{5}$ LMA 22, Boulevard Niels Bohr 69622 - Villeurbanne- Lyon Cedex France;

${ }^{6}$ INFN - Sezione di Napoli and/or Università di Napoli "Federico II" Complesso

Universitario di Monte S. Angelo Via Cintia, I-80126 Napoli, Italia and/or Università di Salerno Via Ponte Don Melillo, I-84084 Fisciano (Salerno), Italia;

${ }^{7}$ Department Artemis - Observatoire de la Côte d'Azur, BP 42209, 06304 Nice Cedex 4, France;

${ }^{8}$ Laboratoire de l'Accélérateur Linéaire (LAL),IN2P3/CNRS-Université de Paris-Sud, B.P. 34, 91898 Orsay Cedex - France;

${ }^{9}$ ESPCI - 10, rue Vauquelin, 75005 Paris - France;

${ }^{10}$ INFN Sezione di Perugia and/or Università di Perugia, Via A. Pascoli, I-06123 Perugia - Italia;

${ }^{11}$ INFN - Sezione di Pisa and/or Università di Pisa, Via Filippo Buonarroti, 2 I-56127 PISA - Italia;

${ }^{12}$ INFN, Sezione di Roma and/or Università "La Sapienza", P.le A. Moro 2, I-00185, Roma.

The Virgo interferometer, aimed at detecting gravitational waves, is now in a commissioning phase. Measurements of its optical properties are needed for the understanding of the instrument. The techniques developed for the measurement of the optical parameters of Virgo are presented in this paper. These parameters are compared to the Virgo specifications.

(C) 2006 Optical Society of America

OCIS codes: $120.2230,120.3180$

\footnotetext{
${ }^{13}$ Corresponding authors: E. Tournefier (tournefier@lapp.in2p3.fr) and F. Bondu (bondu@obs-nice.fr).
} 


\section{Introduction}

Virgo $^{1-3}$ is a gravitational wave antenna aiming at detecting gravitational waves emitted by several types of astrophysical sources. Several other detectors based on the same principle are being operated or commissioned in the USA $\left(\mathrm{LIGO}^{4}\right)$, in Germany $\left(\mathrm{GEO}^{5}\right)$ and in Japan $\left(\mathrm{TAMA}^{6}\right)$. The Virgo detector has been built near Pisa in Italy. It is based on a Michelson interferometer with 3-km long arms. The interferometric detection is based on the following property: as a gravitational wave passes the optical length of one arm gets longer while the other gets shorter. This induces a change in the interference pattern that is detected by measuring the intensity of the light exiting the interferometer output port. ${ }^{7,8}$ In order to improve the sensitivity with respect to a simple Michelson each arm of the interferometer contains a Fabry-Perot cavity $^{9}$ and the power recycling technique ${ }^{9}$ is used with the addition of a partially reflecting mirror at the entrance of the interferometer.

Virgo is in a commissioning phase since Fall 2003. Such a detector has to be kept on an optimal working point by means of quite complex feedback controls and the optical properties of the interferometer have to be well known in order to understand the error signals used for these controls. The optical properties also define the coupling of some of the noises to the gravitational wave signal. This article describes the techniques developed for the measurements of the optical properties of the interferometer. These techniques are applied to the Virgo data to extract its optical properties and compare them with the Virgo design. A detailed simulation is used to interpret these results and to understand which components have to be replaced to achieve the Virgo design sensitivity.

\section{The optical design of Virgo}

The Virgo detector is based on a Michelson interferometer with $3 \mathrm{~km}$ long arms. Figure 1 shows the optical scheme of Virgo. All the mirrors of the interferometer are suspended to a chain of pendulums for seismic isolation. Above the pendulum resonant frequency the mirrors can be considered as free falling masses in the horizontal direction. Each arm contains a Fabry-Perot cavity whose role is to increase the optical path and therefore the phase shift induced by a gravitational wave. To achieve maximal sensitivity the interferometer arm length difference is set to obtain a destructive interference at the interferometer output port. In this condition all the light is reflected toward the laser and it can be reinjected into the interferometer using an additional mirror (the power recycling mirror). This power recycling technique can increase the amount of light impinging on the Michelson beam splitter by orders of magnitude, and as a consequence improves the interferometer sensitivity.

The error signals which are used to control the interferometer globally are based on the Pound-Drever-Hall technique. ${ }^{10,11}$ For this purpose the laser beam is phase modulated at a high (several $\mathrm{MHz}$ ) frequency $f_{\text {mod }}$. This creates additional components to the laser beam 
(the sidebands) whose frequency differs by $n \times f_{\text {mod }}$ ( $\mathrm{n}$ is any integer) from the carrier one. The beams exiting the interferometer are collected on InGaAs photodiodes whose signals are also demodulated at one, two or three times the modulation frequency.

At high frequency the sensitivity of such an interferometer is given by the amplitude of the signal equivalent to the shot noise:

$$
\Delta h_{s n}(f)=\frac{\lambda}{2 \pi L} \sqrt{\frac{h \nu}{2 P_{0}}} \frac{\pi}{2 F} \frac{\sqrt{J_{0}^{2}(m) G(1-C)+6 T J_{1}^{2}(m)}}{2 J_{0}(m) J_{1}(m) \sqrt{G T}} \sqrt{1+\left(\frac{f}{f_{0}}\right)^{2}}
$$

where $\lambda$ is the laser wavelength, $P_{0}$ the input power, $F$ the finesse of the Fabry-Perot cavities and $f_{0}=c / 4 F L$ their cut-off frequency, $G$ the recycling gain of the laser carrier, $T$ the product of the sidebands recycling gain by their transmission to the output port, $1-C$ the contrast defect, $m$ the modulation depth and $J_{0(1)}$ are Bessel functions. The factor 6 in front of $T J_{1}^{2}(m)$ takes into account the fact that the first-order sidebands are not stationary. ${ }^{12}$ Figure 2 shows the shot noise limited sensitivity as a function of the modulation depth for plausible values of the contrast defect and of the transmission of the sidebands. The shot noise limit can be pushed down by optimizing the modulation index for a given contrast defect: the worse the contrast the higher the modulation has to be. Since high modulation depth is difficult to achieve the contrast defect has to be kept as small as possible. For this purpose Virgo uses high quality mirrors ${ }^{13}$ and in addition an output mode cleaner ${ }^{15}$ is used to filter high-order spatial modes that have built up in the interferometer.

The recycling gain is limited by the losses of the Fabry-Perot cavities: the best recycling gain is obtained for a reflectivity of the recycling mirror equal to the reflectivity of the Fabry-Perot cavities. Since the losses are not a priori precisely known, the recycling mirror reflectivity is kept small enough with respect to the expected reflectivity of the long cavities in order to avoid any longitudinal locking instabilities. The transmission factor of the sidebands is also increased with the presence of a recycling cavity, therefore the modulation frequency is chosen such that it resonates inside the recycling cavity.

A particularity of Virgo with respect to other similar interferometers is that the angular alignment of the Fabry-Perot mirrors is based on the Anderson-Giordano technique, ${ }^{16}$ a variant of the Anderson technique: ${ }^{17}$ the modulation frequency used for this purpose is chosen such that when the carrier resonates with the $\mathrm{TEM}_{00}$ mode of the Fabry-Perot cavities the upper sideband frequency coincides with the resonance of the $\mathrm{TEM}_{01}$ mode. It was shown that, provided that the sideband components are resonant in the recycling cavity, a single phase modulation can be used to extract both the longitudinal locking error signals and the alignment error signals. ${ }^{2}$ When the interferometer is on its working point the $\mathrm{TEM}_{00}$ of the carrier resonates in the cavities; this implies that the modulation frequency corresponds to the mode separation frequency of the cavities.

The mode separation frequency (see Section 7.B) is determined by the radius of curvature of 
the mirrors of the cavity and by its length. These quantities also define the size of the beam which resonates in this cavity. The input beam parameters have therefore to be matched to these properties.

The Virgo optical design characteristics are: $F=50, G=50, T=0.46$, flat Fabry-Perot input mirrors and curved end mirrors with a radius of curvature $R_{\text {end }}=3450 \mathrm{~m}$, and the input beam waist $w_{0}=2 \mathrm{~cm}$. All mirrors were required to have losses lower than $10 \mathrm{ppm} .^{2}$ The modulation frequency was set at $f_{\text {mod }}=6.26 \mathrm{MHz}$.

The mirror reflectivities have been defined by the gain of the cavities ( $F$ and $G$ ). After the coating, the reflectivity and the mirror surface profile have been measured before installation on the Virgo site. These mirror maps are incorporated in an optical simulation, described in Section 3, in order to obtain a realistic estimation of the Virgo optical parameters. These parameters are also deduced from the interferometer signals. The measurements are reported in the following sections and compared to simulation results when it is relevant. Concerning the laser beam parameters, the measurement of the modulation index and the tuning of the modulation frequency are described in Section 4. Section 5 describes the input mode cleaner measurements while the matching of the input and output beams are discussed in Section 6 . The measurement of the characteristics of the Fabry-Perot and of the recycling cavities are described in Sections 7 and 8 respectively. Finally the contrast defect is discussed in Section 9 .

The measurements reported in this paper have been performed with the 2005 optical configuration which does not include the replacement of the power recycling mirror and the modifications on the injection system introduced at the end of 2005.

\section{Simulation of Virgo optical properties}

The SIESTA ${ }^{21}$ simulation has been used to produce time domain signals which, once compared to real data signals allow the extraction of some optical parameters, like the finesse of the cavities.

In addition, we have developed two independent simulation codes that both aim at testing the effects of interferometer defects on its overall sensitivity. Real interferometer characteristics that can be studied are typically beam and mirrors misalignments, length mismatch, mirror defects, thermal deformations and so on. The simulation results can be sensitive to the way these characteristics are represented so that two independent codes proved to be very useful. Both simulations are static, so only steady state fields are computed.

One code (DarkF) is based on the representation of the various fields in a plane wave basis and on a fast Fourier transform algorithm for propagation, while in the second code (NV) the fields are represented in an Hermite-Gauss functions basis. DarkF and NV don't have exactly the same properties. For the simulations with some centered defects at long correlation 


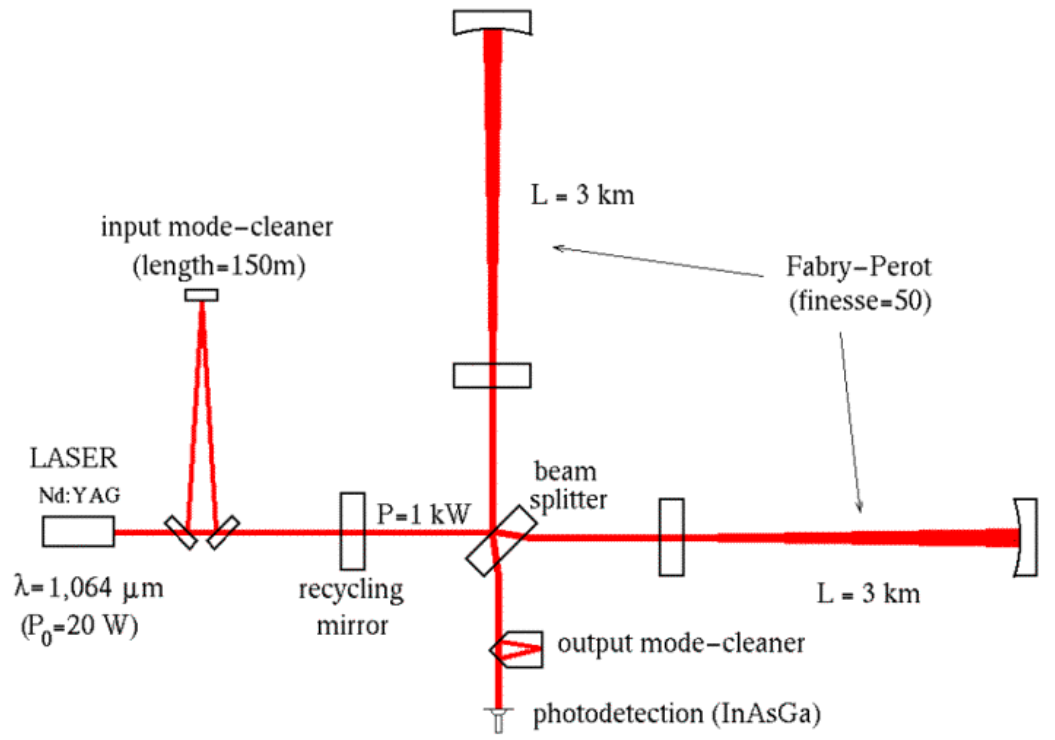

Fig. 1. Optical scheme of Virgo.

length (typically of the order of the centimeter), NV is well adapted. DarkF is also adapted to the simulations with defects at short correlation length (but larger than the sampling step).

\section{A. The DarkF simulation}

DarkF is an optical simulation code using a plane wave decomposition to propagate the wavefronts. Beams and mirrors are sampled on a grid (x,y) and the DarkF resolution depends on the grid size and on the number of points. Typically a size of $0.7 \mathrm{~m}$, i.e. larger than a mirror and 128x128 points are used. This granularity is good enough since the results do not change when the number of points is increased. With the Fourier transform method, the propagated beam $\mathrm{E}_{2}(\mathrm{x}, \mathrm{y}, \mathrm{z})$ is obtained from the initial beam $\mathrm{E}_{1}\left(\mathrm{x}, \mathrm{y}, \mathrm{z}_{0}\right)$ and a propagator $(\mathrm{P})$ in the Fourier space ( $\mathrm{z}$ is the propagation axis): ${ }^{18,19}$

$$
\begin{aligned}
E_{2}(x, y, z) & =F T^{-1}\left(F T\left(E_{1}\left(x, y, z_{0}\right)\right) \times e^{-i \frac{p^{2}+q^{2}}{2 k}\left|z-z_{0}\right|}\right) \\
& =F T^{-1}\left(F T\left(E_{1}\left(x, y, z_{0}\right)\right) \times P\right)
\end{aligned}
$$

where $F T$ and $F T^{-1}$ are the Fourier and inverse Fourier transforms respectively and $p$ and $q$ are the Fourier space coordinates. This code can be used to simulate a simple or a complex system as a Fabry Perot cavity or Virgo. To obtain an intracavity beam (Ecav) composed 


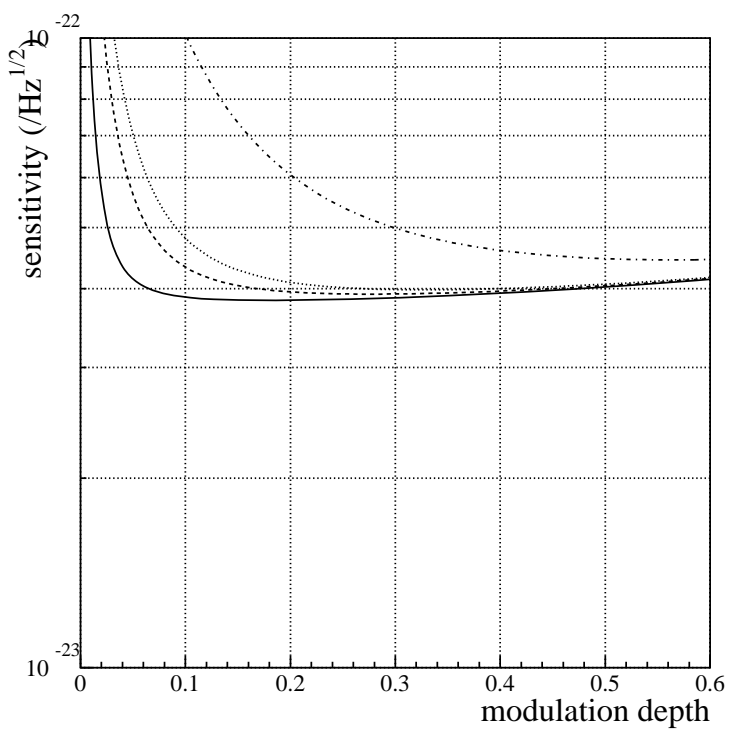

Fig. 2. Shot noise limited sensitivity as a function of the modulation depth for several values of sidebands transmission $(T)$ and contrast defect $(1-C)$ : $T=0.4,1-C=10^{-5}$ (continous line), $T=0.2,1-C=3 \times 10^{-5}$ (dashed line), $T=0.1,1-C=3 \times 10^{-5}$ (dotted line) and $T=0.1,1-C=3 \times 10^{-4}$ (dash-dotted line). 
by two mirrors $M_{1}$ and $M_{2}$ a convergence algorithm is used:

$$
\operatorname{Ecav}_{n+1}=t_{1} E_{0}-r_{1} r_{2} M_{1} P_{L} M_{2} P_{L} \operatorname{Ecav}_{n}
$$

where $P_{L}$ is the propagator on a length $\mathrm{L}, r_{1}$ and $r_{2}$ the reflection coefficient of $M_{1}$ and $M_{2}$, $t_{1}$ the transmission coefficient of $M_{1}$, and $E_{0}$ the incoming beam. The main drawback of DarkF is its large computing time (typically of the order of one hour for the configurations used here).

\section{B. The NV simulation}

In the Hermite-Gauss representation each interface, substrate, or free space is represented by a complex operator, calculated in the Hermite-Gauss space, which acts on the field incident upon it. Starting from real cartesian maps of the mirrors surface $z(x, y)$ and reflectance $r(x, y)$ taken from metrology instruments, elementary reflexion operators $r_{i, j \rightarrow m, n}$ are calculated using :

$$
r_{i, j \rightarrow m, n}=\int_{x, y} g_{i, j}(x, y) r(x, y) \exp (4 i \pi z(x, y)) g_{m, n}^{*}(x, y) d x d y
$$

where the $g$ 's are the Hermite-Gauss basis functions, and $g^{*}$ denotes their complex conjugate. Transmission operators are calculated in the same way. The elementary operators can then be combined to create operators representing whole optical components, Fabry-Perot cavities, and eventually the full interferometer. Those operators are applied on the field $u$ represented as a superposition of Hermite-Gauss modes:

$$
u(x, y)=\sum_{i, j} u_{i j} g_{i, j}(x, y)
$$

where the sum runs over all the basis functions. The field $u^{\prime}$ transformed after interaction with an optical component is then simply calculated by applying the relevant operator on the incident field like :

$$
u_{m n}^{\prime}=\sum_{i, j} r_{i, j \rightarrow m, n} u_{i j}
$$

Figure 3 shows the intensity reflexion operator of Virgo, calculated using maps for each optical component. As can be seen on the figure, working with an Hermite-Gauss basis gives immediately access to all the coupling factors between $\mathrm{TEM}_{i j}$ modes inside Virgo and their precise localization, and allows the immediate computation of the energy in each mode everywhere in the interferometer and outside. The main limitation of this particular code is the difficulty to represent accurately beams with shapes that strongly differ from that of a $\mathrm{TEM}_{00}$ beam without using a prohibitive number of Hermite-Gauss functions. In order to test these effects at least 153 basis functions are used. 


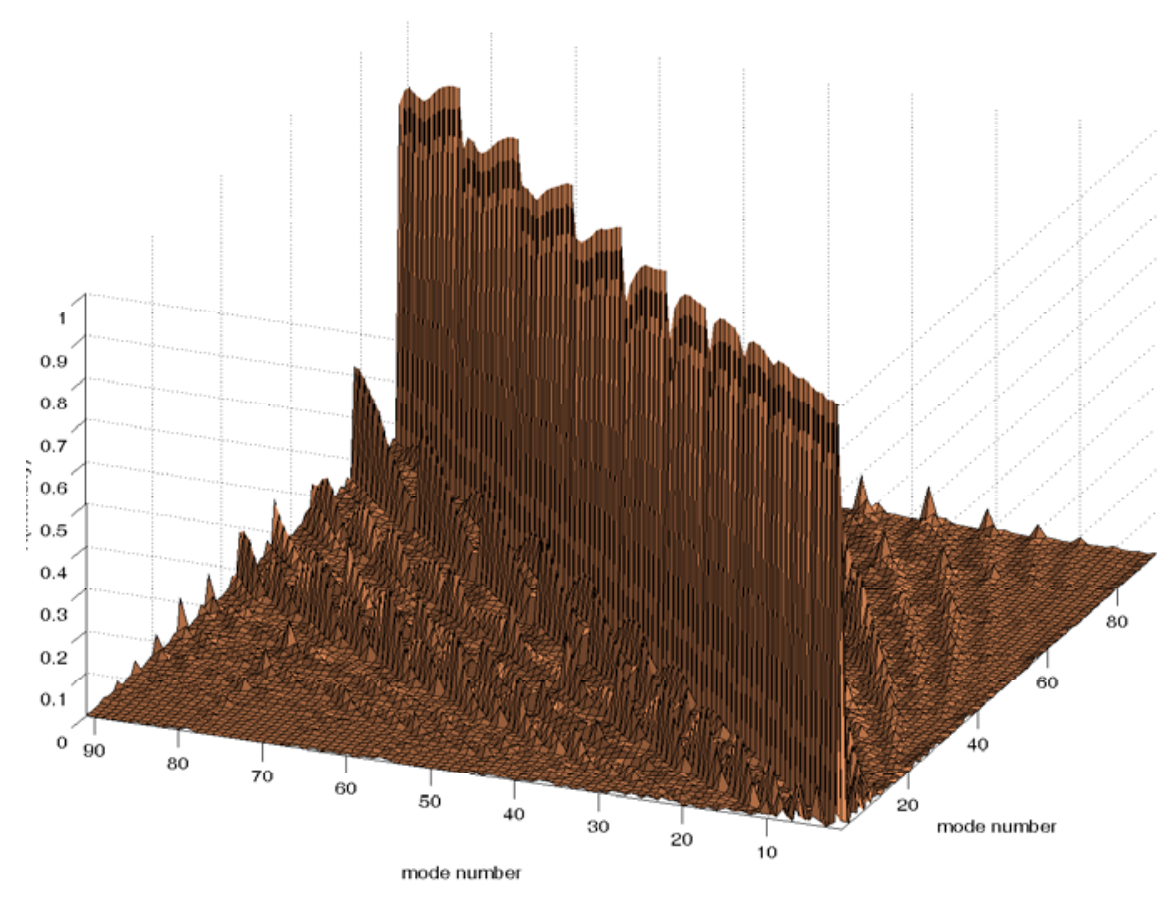

Fig. 3. Intensity reflection operator of the full interferometer with real maps. The modes are ordered $\{00,01,10,02,11,20,03, \ldots\}$. Off-diagonal elements show coupling between modes inside the interferometer induced by surface defects. Diagonal elements slightly differ from unity mainly due to the finite size of the coatings. 


\section{Modulation of the laser beam}

The laser beam is phase modulated by mean of an electro-optic modulator (EOM) located before the input mode cleaner. The value of the modulation depth is set by varying the voltage applied on the modulator and its value is cross-checked with the interferometer signals. The modulation frequency has to be carefully tuned so that it fulfills the required resonance conditions inside the optical cavities of the interferometer.

\section{A. Modulation depth measurement}

The modulation depth has first been set to a small value, around 10\%, and will be increased in the future if it is needed to improve the sensitivity. The value set at the level of the modulator is cross-checked with the interferometer data in the following ways:

1- A calibration of the voltage applied on the EOM was performed for a high voltage value ( $\sim 5$ Volts), using signals from a reference cavity located before the input mode cleaner. It is based on the same principle as the third method described below. An extrapolation of this calibration to the applied voltage gives $m=0.13 \pm 0.03$.

2- With the signals observed with the central part of the interferometer aligned and free mirrors: the amplitude of the demodulated signal is proportional to $J_{0}(m) J_{1}(m)$ while the amplitude of the DC signal is proportionnal to $J_{0}(m)$. The ratio of the amplitudes of these signals gives therefore access to the value of $\mathrm{m}: m=0.16 \pm 0.01$ where the uncertainty comes from the knowledge on the calibration of the signals.

3- With a free Fabry-Perot cavity, using the measurement of the power stored inside the cavity for the sidebands and the carrier. When the $\mathrm{TEM}_{00}$ mode of the carrier resonates the power is proportional to $J_{0}^{2}(m)$ while when the $\mathrm{TEM}_{00}$ mode of the upper sideband resonates it is proportional to $J_{1}^{2}(m)$. These resonances are shown in Figure 10. In this case the ratio of these signals is free of any calibration errors since the same photodiode channel is used and is just limited by the noise on the small sideband signal. The measurement gives $m=0.165 \pm 0.005$.

Both methods based on the interferometer signal agree well and the value obtained is used in the following.

\section{B. Modulation frequency}

Since the beam is modulated before the input mode cleaner (IMC) the sidebands should resonate in the IMC in order to be transmitted. As was pointed out in Section 2 they should also resonate inside the recycling cavity and correspond to the Anderson frequency (i.e. the frequency spacing between the resonance of $\mathrm{TEM}_{01}$ mode and the resonance of 
the $\mathrm{TEM}_{00}$ mode) of the Fabry-Perot cavities. The radius of curvature of the end mirror defines the mode separation frequency of the cavity. This gives a set of possible values for the modulation frequency. Given the foreseen range of values for the input mode cleaner length and the recycling cavity length, the value of the modulation frequency has been set, among the possible values which matched the resonance condition inside these cavities, to 6.26 MHz. The fine tuning is then done after the precise measurement of the mode cleaner length. The modulation frequency has to be an exact multiple of the IMC's free spectral range:

$$
f_{\text {mod }}=n \mathrm{FSR}_{\mathrm{IMC}}=n \frac{c}{2 L_{\mathrm{IMC}}},
$$

with $n=6$. This condition has to be tuned very carefully since any mismatch couples the length noise of the IMC to the interferometer signals used for its longitudinal controls. After the measurement of the IMC length (see Section 5) the modulation frequency has been adjusted to $f_{\text {mod }}=6.264180 \mathrm{MHz}$.

It has then been checked that it matched the recycling length and the mode separation frequency:

- The condition of resonance inside the recycling cavity takes into account the phase shift introduced by the Fabry-Perot cavities. An analytical calculation and simulations with SIESTA ${ }^{21}$ and Finesse ${ }^{22}$ give the same optimal recycling length: $l_{\text {rec }}=12.073 \mathrm{~m}$ for this modulation frequency. This condition should be fulfilled within about $1 \mathrm{~cm}$ in order not to degrade the recycling factor. The length of the recycling cavity has been measured (see Section 8.A.2) and agrees with this requirement within $1 \mathrm{~cm}$.

- The Anderson technique, used for the angular alignment of the Fabry-Perot cavities, requires that the TEM01 mode of the upper sideband is distant from the carrier by a multiple of the free spectral range, and therefore the modulation frequency should fulfill:

$$
f_{\mathrm{mod}}=f_{\mathrm{sep}}+n \mathrm{FSR}_{\mathrm{FP}}
$$

where $f_{\text {sep }}$ is the mode separation frequency of the Fabry-Perot cavity and FSR $\mathrm{FP}_{\text {is }}$ its free spectral range. The Anderson technique requires that the modulation frequency corresponds to the optimum frequency given by Equation (5) within the linewidth of the cavity $(1000 \mathrm{~Hz})$. The mode separation frequency is deduced from the measurement of the end mirror radii of curvature (see Section 7.B) and the free spectral range has been measured as described in $7 . \mathrm{C}, \mathrm{FSR}_{\mathrm{FP}}=(49967 \pm 1) \mathrm{Hz}$. The optimal values of $f_{\text {mod }}$ are therefore (with $\left.n=125\right): f_{\text {mod }}^{\text {opt } \mathrm{N}}=(6264420 \pm 100) \mathrm{Hz}$ for the north cavity and $f_{\text {mod }}^{\text {opt, }}=(6264340 \pm 200) \mathrm{Hz}$ for the west cavity. The value set for the modulation frequency corresponds to the optimum values within at most $300 \mathrm{~Hz}$ and is therefore well within the allowed range. 
Following these measurements it can be concluded that all the required resonance conditions for the sidebands are fulfilled.

\section{The input mode cleaner cavity}

The input mode cleaner (IMC) is a triangular optical cavity whose aim is to filter the input beam jitter and to obtain a clean gaussian beam at the entrance of the interferometer. Its optical components are suspended to achieve adequate seismic isolation. Therefore it offers a good reference for the laser frequency and is used to stabilize the laser frequency. The mode cleaner design parameters are: a length of $143 \mathrm{~m}$, a finesse of 1000, and a waist of $5 \mathrm{~mm}$. To achieve these goals the mirrors of the cavity have been designed with the following characteristics: a radius of curvature of $(183 \pm 5) \mathrm{m}$ for the end mirror and a transmittance of $(3000 \pm 300)$ ppm for both the input and output mirrors.

The measurement of the input mode cleaner optical properties are detailed in the following.

\section{A. The input mode cleaner transmittance}

The transmittance of the IMC is degraded by losses inside the cavity and by the imperfect matching of the input beam to the cavity.

\section{A.1. Losses of the input mode cleaner}

The transmittance of the cavity for the $\mathrm{TEM}_{00}$ mode can be deduced from the measurements of the individual transmittance of the mirrors and of their losses. The DC transmittance of the cavity is, according to Equation (6) of, ${ }^{20}$

$$
T=\frac{4 T_{1} T_{2}}{\left(T_{1}+T_{2}+L_{\mathrm{RT}}\right)^{2}}
$$

where $T_{1}$ and $T_{2}$ are the input and output mirror transmittances, and $L_{\mathrm{RT}}$ the round trip losses, including the far curved mirror transmittance. The input and output mirror transmittances have been measured after coating: $T_{1}=(2427 \pm 30) \mathrm{ppm}$ and $T_{2}=(2457 \pm 30)$ ppm.

The measurement of the cavity pole $f_{P}$ and of the free spectral range FSR are necessary to compute the round trip losses $L_{\mathrm{RT}}$ which can be deduced from the equation:

$$
T_{1}+T_{2}+L_{\mathrm{RT}}=4 \pi \frac{f_{P}}{\mathrm{FSR}} .
$$

The free spectral range and the cavity pole are measured with the open loop transfer function $H$ of the lock of the laser on the cavity around the free spectral range frequency. In this frequency range, the shape is given by the transfer function $H$ between frequency noise and Pound-Drever-Hall signal. The result is fitted with

$$
y=A \exp (\phi)\left(\frac{1+i(f-\mathrm{FSR}) / f_{Z}}{1+i(f-\mathrm{FSR}) / f_{P}}\right)
$$



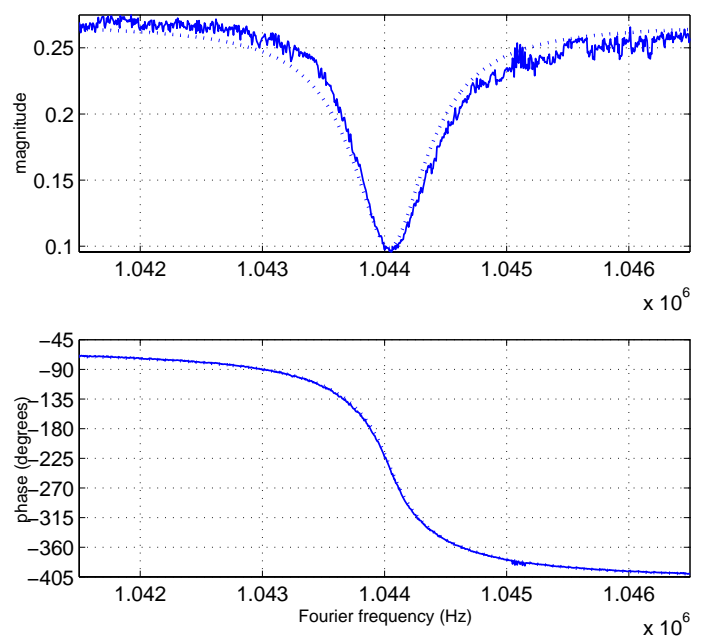

Fig. 4. Open loop transfer function of the lock of laser frequency on the cavity, around $1 \mathrm{MHz}$. In blue, measured data; in red, the fitted curve.

similar to Equation (18) in, ${ }^{20}$ where $A$ and $\phi$ are arbitrary amplitude and phase of the open loop transfer function at this frequency, and $f_{Z}$ an empirical zero which is needed to fit the data and reflects a not understood experimental effect. The fit, shown in Figure 4, gives with 95\% confidence level errors: $f_{P}=(479 \pm 3.3) \mathrm{Hz}$ and FSR $=(1044039 \pm 2.2) \mathrm{Hz}$. The losses deduced from these values, using Equation $(7)$ are $L_{\mathrm{RT}}=(886 \pm 60) \mathrm{ppm}$.

An alternative way to measure the cavity pole $f_{P}$ is the determination of the decay time. It is measured after a fast change of the laser frequency, large enough to drive the cavity out of resonance. A fit of the measured data with an exponential decay, shown in Figure 5, gives a measurement of the decay time: $\tau=(167.2 \pm 0.2) \mu$ s. Then, using $f_{P}=1 /(4 \pi \tau)=$ $(475.9 \pm 0.6) \mathrm{Hz}$ and Equation $(7)$ the total losses are deduced: $L_{\mathrm{RT}}=(846 \pm 45) \mathrm{ppm}$ (where the uncertainty is dominated by the uncertainties on $T_{1}$ and $T_{2}$ ). This is in agreement with the value measured with the transfer function.

Then the expected transmission of the input mode cleaner for the $\mathrm{TEM}_{00}$ mode is $T=$ $(73 \pm 2) \%$ (from Equation (6)).

\section{A.2. Beam matching and radius of curvature of the end mirror}

The beam matching is deduced from the measurement of the transfer function $H$ around the frequency of resonance of the $\mathrm{TEM}_{02}$ mode. The result is fitted with

$$
y=A \exp (\phi)\left(1-\frac{M}{2} \frac{1}{1+i\left(f-f_{02}\right) / f_{P}}\right)
$$




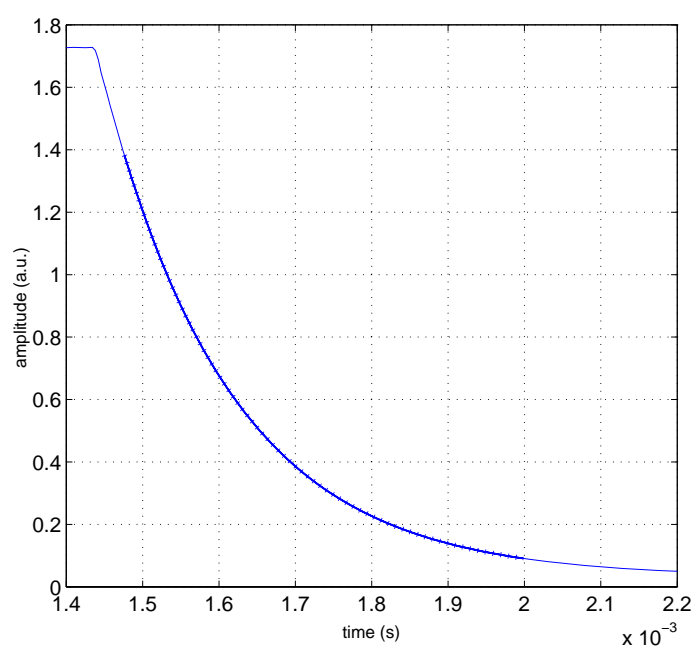

Fig. 5. Time decay experiment: power transmitted by the input mode cleaner, after a fast change of input laser frequency

from Equation (21) in, ${ }^{20}$ where $A$ and $\phi$ are arbitrary amplitude and phase of the open loop transfer function at this frequency, $M$ is the amount of light intensity coupled to the $\mathrm{TEM}_{02}$ mode of the cavity, $f_{02}$ is the frequency of the $\mathrm{TEM}_{02}$ mode, $f_{P}$ is the pole of the cavity. The fit gives, with $95 \%$ confidence intervals: $f_{P}=(593 \pm 51) \mathrm{Hz}, M=0.17 \pm 0.01$, $f_{02}=(726569 \pm 35) \mathrm{Hz}$. The value of the pole is out of the boundaries given by previous measurements, probably because this measurement is noisier as can be observed from Figure 6.

Thus, the mode shaping mismatching is $17 \%$. A mismatching of about $15 \%$ had also been deduced from the measurement of the size of the beam which confirms the measurement presented here.

The effective radius of curvature $R$ can also deduced from the measurement of the frequency of the $\mathrm{TEM}_{02}$ mode $f_{02}$ (as explained in the case of the Fabry-Perot cavities in Section 7.B). The deduced value is $R=(181.99 \pm 0.01) \mathrm{m}$, in agreement with the design specification.

\section{A.3. Total transmittance of the input mode cleaner}

The measurement of the power of the beams at the output ports of the interferometer can be used to deduce the power incident on the interferometer. The values found range from 7 to $8 \mathrm{~W}$.

The measurements described in the previous sections lead to an expected total transmittance 

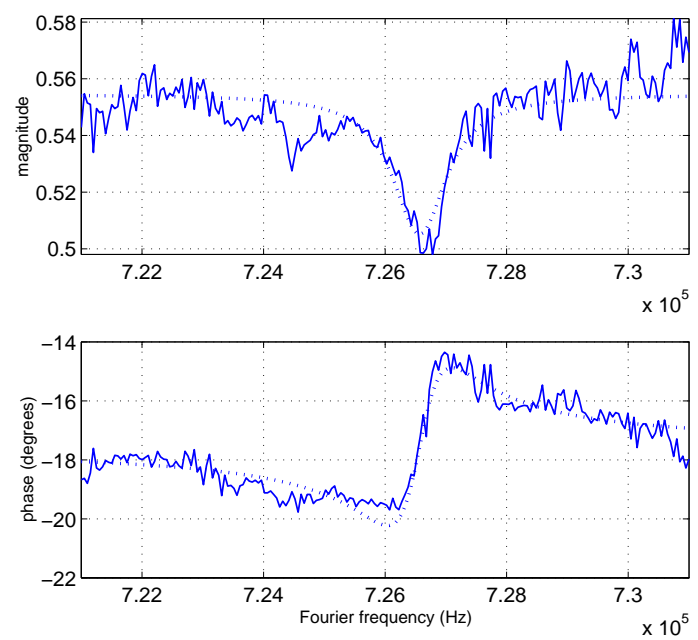

Fig. 6. Open loop transfer function of the lock of laser frequency on the cavity, around the frequency of the TEM $M_{02}$ mode (1726 kHz). In blue, measured data; in red, the fitted curve.

of the input mode cleaner of ( $60 \pm 3) \%: 83 \%$ due to imperfect beam matching and $73 \%$ due to the transmittance of the $\mathrm{TEM}_{00}$ mode. The input power being $16.7 \mathrm{~W}$, the power transmitted by the output mode cleaner is therefore expected to be $10 \mathrm{~W}$. The difference with the power incident on the interferometer points to additional losses which have not been understood yet.

\section{B. Finesse and length of the input mode cleaner}

The other optical properties of the cavity are also deduced from the transfer function measurements and the decay time experiment. The finesse is directly deduced from the measurements of the pole of the cavity $f_{P}$ described in the above sections:

$$
F=\frac{c}{4 f_{P} L_{\mathrm{IMC}}}
$$

The most precise measurement comes from the decay time experiment and gives $F=1097 \pm 2$. The optical length of a half round trip is deduced from the measurement of the free spectral range:

$$
\mathrm{FSR}=\frac{c}{2 L_{\mathrm{IMC}}}
$$

and gives $L_{\mathrm{IMC}}=(143533326 \pm 30) \mu \mathrm{m}$. 


\section{Matching of the input and output beams}

Any mismatch of the beam with respect to the optical cavities of Virgo (input and output mode cleaners and Fabry-Perot cavities) results into a loss of the effective power and therefore a worsening of the sensitivity. Some loss of the input power is also the result of a nonunity transmittance of the input mode cleaner. The tuning of the beam parameters and the measurement of these losses are described in this section.

\section{A. Input beam matching to the interferometer}

The beam exiting the input mode cleaner has a waist of $5 \mathrm{~mm}$ and is adjusted to the Virgo Fabry-Perot cavities by means of an off-axis telescope formed by two spherical mirrors. The Virgo beam waist is around $20 \mathrm{~mm}$ and is located at the Fabry-Perot input mirrors. The length of this telescope has to be tuned so that the beam incident on the interferometer matches as well as possible the fundamental mode of the Fabry-Perot cavities. The tuning consists in adjusting the distance between the two mirrors forming the input telescope in order to obtain the highest power stored inside the Fabry-Perot cavities at the $\mathrm{TEM}_{00}$ resonance.

The size of the beam on the end mirrors during the tuning of the telescope is compared to a simulation with Finesse ${ }^{22}$ as shown in Figure 7. This allows a rough extraction of the input beam parameters: $\omega_{x}=18 \mathrm{~mm}, z_{0 x}=-500 \mathrm{~m}, \omega_{y}=18 \mathrm{~mm}, z_{0 y}=500 \mathrm{~m}$ where the $\mathrm{z}$ axis is oriented towards the injection system and its origin is located on the beam splitter mirror. After this tuning it is expected that about $94 \%$ of the input beam is coupled to the $\mathrm{TEM}_{00}$ mode of the cavities. This coupling is measured by comparing the amplitude of the first Laguerre mode to the amplitude of the $\mathrm{TEM}_{00}$ mode in the Fabry-Perot cavities (see Figure 10) and is found to be $94 \%$. This could not be further improved since the beam was astigmatic due to a bad centering of the beam on the spherical mirrors of the telescope: a better alignment of the beam would have been needed.

\section{B. Matching of the beam to the output mode cleaner}

The beam entering the output bench has a waist of about $2 \mathrm{~cm}$. The dark fringe beam is passed through the output mode cleaner (OMC) in order to improve the contrast defect. The waist of the mode cleaner cavity is $140 \mu \mathrm{m}$. The output beam is adapted to the mode cleaner cavity by means of a telescope made of three lenses. The position of these lenses

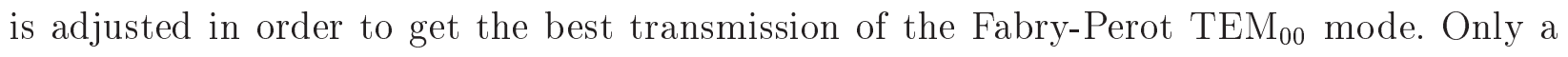
rough matching was done using the direct beam exiting from the injection system. Due to the astigmatism of this beam the matching to the output mode cleaner could not exceed $94 \%$. The relative position of the lenses is tuned in order to minimise the power reflected by the $\mathrm{OMC}$ and a matching of $93 \%$ is reached. 


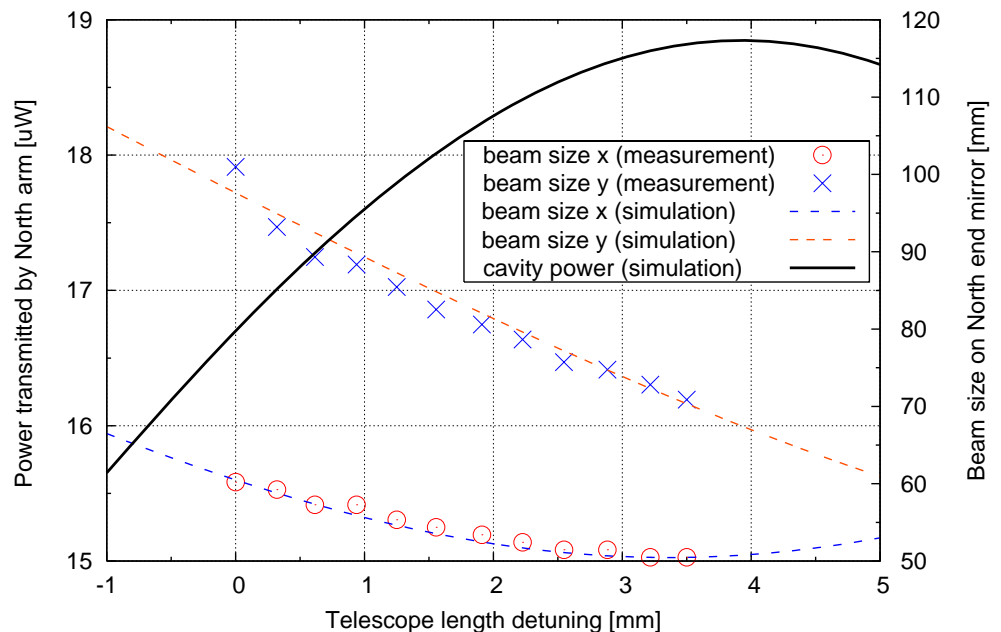

Fig. 7. Size of the beam after $3 \mathrm{~km}$ along two orthogonal axis as a function of the input telescope length tuning. The curves represents the result of the simulation while the dots are the experimental measurements. The wider black curve shows the power transmitted by the cavity as predicted by simulation. ${ }^{22}$

The matching is then cross-checked with the beam reflected by the North Fabry-Perot cavity. The cavity is kept at resonance and the end mirror position is excited with a frequency line. This line should be seen only by the mode which resonates into the cavity, i.e. the FabryPerot $\mathrm{TEM}_{00}$ mode. Therefore, comparing the amplitude of this line on the beams reflected and transmitted by the OMC gives an estimate of the OMC transmission for the FabryPerot $\mathrm{TEM}_{00}$. It is found to be around $94 \%$ and could in principle be improved with a better tuning of the telescope. The matching was considered to be good enough at that time and no further tuning was performed. It will be repeated when the Virgo sensitivity gets closer to its design goal.

\section{Fabry-Perot cavities}

The Fabry-Perot cavities are characterized by their finesse, their length, the radius of curvature of the end mirrors and the losses due to defects of the mirrors. This section reports on the measurement of these quantities.

\section{A. Finesse}

The finesse of the Fabry-Perot cavities is extracted from the TEM $_{00}$ Airy peaks: it is defined as the ratio of the distance between two consecutive $\mathrm{TEM}_{00}$ resonances (FSR) to their 
linewidth (FWHM). Unfortunately, due to dynamical effects the Airy peak is distorted, as shown in Figure 8, and this effect prevents measuring the linewidth directly. Nevertheless, if the speed of the cavity mirrors is known this distortion can be predicted by a simulation including dynamical effects. It is therefore first needed to measure the speed of the cavity, then the shape of the Airy peak is compared to a time domain simulation including dynamical effects $\left(\right.$ SIESTA $\left.^{21}\right)$ for a set of finesse values.

The speed of the cavity can be determined from the measurement of the cavity length as a function of time using the fact that the cavity length difference between two $\mathrm{TEM}_{00}$ resonances is equal to $\lambda / 2$. If the cavity is not too much excited (angularly and longitudinally) its length varies sinusoidally with time. Figure 9 shows the length of the cavity as a function of time and the cosine function fitted to these points: $l(t)=\cos (\omega t+\phi)$. The speed is derived from $l(t)$ and is measured with an uncertainty of 1 to $2 \%$.

For each Airy peak the simulation is run with the measured speed for a set of finesse values. The shape of the peak is compared to these simulations and the value which best matches the data is kept. This measurement is repeated on several points and the dispersion of these measurements defines the accuracy. Table 1 gives the measurement for each cavity as well as the values expected from the mirror coating measurements:

$$
F=\frac{\pi \sqrt{r_{1} r_{2}}}{1-r_{1} r_{2}}
$$

where $r_{1(2)}$ is the field reflectivity of the input (end) mirrors. It should be noted that since the input mirror is flat, its effective reflectivity is expected to vary due to thickness variation induced by temperature and therefore the finesse of the cavity is expected to vary as well. This effect is proportional to the value of the second face anti-reflective coating of the input mirror: $\Delta F / F= \pm 2 r_{A R}$ where $r_{A R}$ is the field reflectivity of this second face. The coating measurement gave $R_{A R}=150 \mathrm{ppm}$ and therefore the variation of finesse are expected to be about $\pm 2.5 \%$.

Table 1. Fabry-Perot finesses

\begin{tabular}{|c|c|c|}
\hline & Measurement & From mirror reflectivities \\
\hline North FP cavity & $49.0 \pm 0.5$ & 50 \\
\hline West FP cavity & $51 \pm 1$ & 51 \\
\hline
\end{tabular}

The values of the two Fabry-Perot finesses are obtained with the measurement based on the shape of the Airy peaks (first column) compared to those deduced from the mirror reflectivity measurements (second column). 


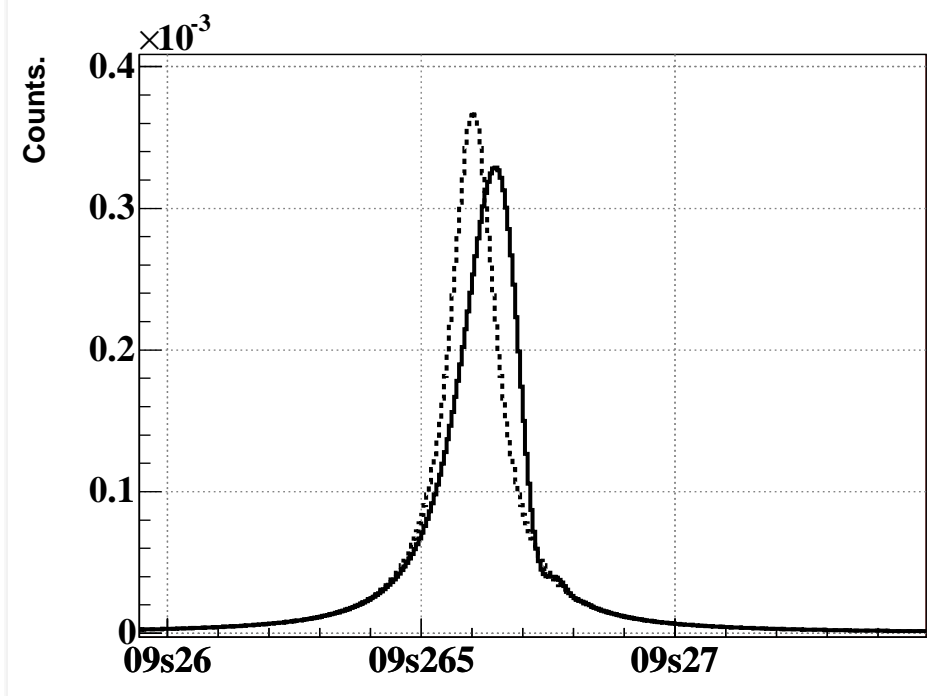

Fig. 8. Profile of the Airy peak without dynamical effects (dotted line) and with dynamical effects (full line) for a speed of $10 \mu \mathrm{m} / \mathrm{sec}$.

7.B. Radius of curvature of the end mirrors and mode separation frequency

The radius of curvature of the end mirrors defines the waist of the beam but, as shown in Section 4.B, it is also related to the modulation frequency, via the mode separation frequency of the cavities. The mode separation frequency $f_{\text {sep }}$ corresponds to the frequency difference for which the $\mathrm{TEM}_{00}$ and the $\mathrm{TEM}_{01}$ modes resonate and is related to the radius of curvature of the end mirror $R_{2}$ (the input miror is flat) and the length of the cavity $L_{F P}$ :

$$
f_{\mathrm{sep}}=\nu_{01}-\nu_{00}=\frac{c}{2 \pi L_{\mathrm{FP}}} \operatorname{acos} \sqrt{1-\frac{L_{\mathrm{FP}}}{R_{2}}}
$$

The radius of curvature can be deduced from the signals measured at the transmission of the Fabry-Perot cavities and also from the measurement of the map of the mirrors as described in the following. The mode separation frequency also depends on the cavity length whose measurement is described in 7.C. 


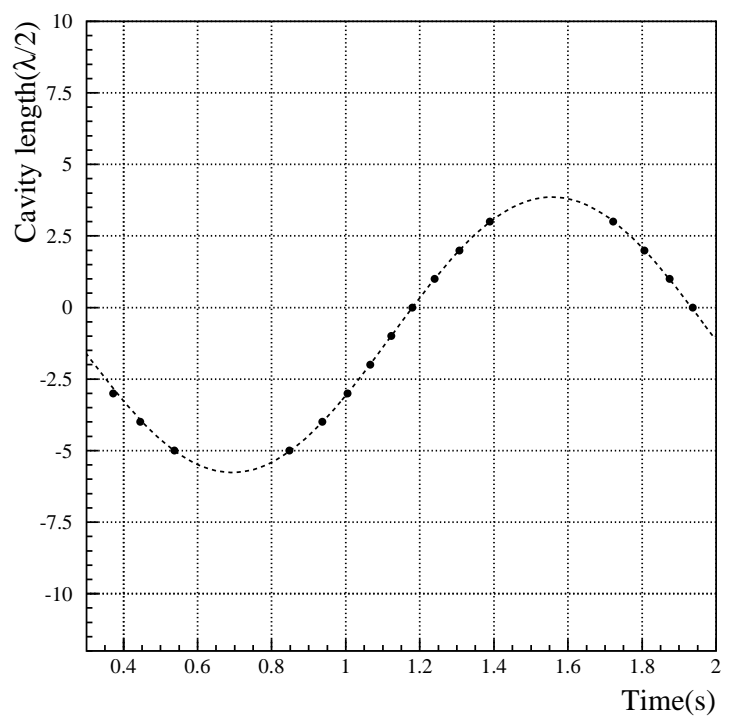

Fig. 9. Example of the measurement of the speed of the Fabry-Perot cavities: the dots represent the TEM $M_{00}$ peak positions and the line shows the length $l(t)$ fitted to these points. 


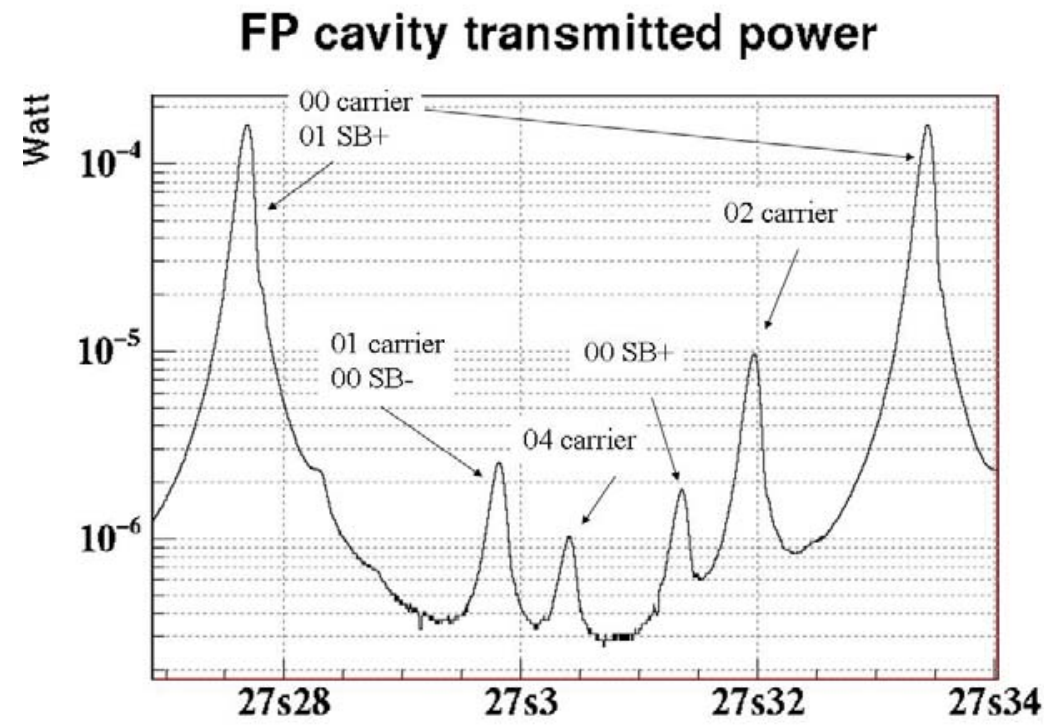

Fig. 10. Power transmitted by a free Fabry-Perot cavity.

\section{B.1. Measurement with the Fabry-Perot signals}

The radius of curvatures can be deduced from the optical spectrum of the cavity by measuring the cavity length difference between the $\mathrm{TEM}_{n m}$ modes:

$$
\frac{d_{n m-00}}{(n+m) d_{00-00}}=\frac{1}{\pi} \mathrm{acos} \sqrt{1-\frac{L_{\mathrm{FP}}}{R_{2}}}
$$

where $d_{n m-00}$ is the length difference between the resonances of $\mathrm{TEM}_{n m}$ and $\mathrm{TEM}_{00}, d_{00-00}$ is the length difference $(\lambda / 2)$ between two consecutive $\mathrm{TEM}_{00}$ resonances.

Figure 10 shows the transmitted power when the cavity is freely swinging and well aligned. One can recognise the $\mathrm{TEM}_{n m}$ resonances for low values of $m+n$. The modes of the carrier are used to do this measurement. Since the $\mathrm{TEM}_{00}$ of the lower sideband is superimposed to the $\mathrm{TEM}_{01}$ of the carrier the cavity has to be enough misaligned so that the $\mathrm{TEM}_{01}$ of the carrier is dominant. The data used for this measurement fullfil this criterion. The cavity length is reconstructed as a function of time with the same method as described in Section 7.A. The length difference between the modes can then be reconstructed. The measurement is done for $m+n=1,2,4$ and uses several tens of points. The uncertainty on $d_{n m-00}$ is given by the RMS of its distribution. The effective radius of curvature is deduced from formula (14). The effective radius of curvature extracted from these measurement are given in Table 2. The precision is typically of the order of 20 meters. 
Since the mirror curvature is not necessarily uniform different modes might see a different radius of curvature since they have different distribution of power. The radii of curvature seen by different modes are compatible showing that the mirror curvature is reasonably uniform.

\section{B.2. Measurement from the mirror surface maps}

The surface maps of the mirrors have been measured after coating. The radius of curvature can be deduced from these maps in several ways:

(1) with a simulation of the FP cavity including the maps based on FFT field propagation (DARKF) and applying the same method as on real data

(2) with the same simulation as in (1) but using the size of the beam which resonates in the cavity since it is directly related to the radius of curvature of the cavity mirrors

(3) by fitting the map of the mirrors with a sphere weighted by a gaussian in order to simulate the beam profile.

The radius of curvature determined with these three methods are given in Table 2.

The measurements of the radius of curvature based on the Fabry-Perot signals agree with the results based on the mirror surface maps for the North cavity but are systematically about $70 \mathrm{~m}$ lower for the West cavity. It is now known that a small bias was introduced during the measurement of these maps due to the apparatus holding the mirror. This could explain this difference.

\section{B.3. Mode separation frequencies}

These results are turned into a measurement of the mode separation frequency using Equation (13). Since the Anderson technique used in Virgo is based on the $\mathrm{TEM}_{01}$ mode only results from this mode are used. The Anderson frequencies are:

- for the North arm: $f_{\text {sep }}^{\text {north }}=(18533 \pm 100) \mathrm{Hz}$

- for the West arm: $f_{\text {sep }}^{\text {west }}=(18450 \pm 200) \mathrm{Hz}$.

These values are compared to the modulation frequency in Section 4.B.

\section{C. Arm length}

The length of the arms can be extracted with a method similar to that described for the measurement of the radius of curvature in section 7.B by noting that the difference of length between the $\mathrm{TEM}_{00}$ resonance of the sidebands and of the carrier corresponds to the modulation frequency modulo the free spectral range:

$$
f_{\text {mod }}=\nu_{\mathrm{USB}}-\nu_{\text {carrier }}+125 \mathrm{FSR}=\frac{c}{2 L_{\mathrm{FP}}}\left(\frac{d_{\mathrm{USB}-\text { carrier }}}{d_{00-00}}\right)+125 \mathrm{FSR}
$$


Table 2. Radius of curvature of the end mirrors.

\begin{tabular}{|l|c|c|c|c|}
\hline & interferometer data & Mirror maps (1) & Mirror maps (2) & Mirror maps (3) \\
\hline North cavity & $\mathrm{m}+\mathrm{n}=13555 \pm 20$ & $\mathrm{~m}+\mathrm{n}=13563 \pm 20$ & & \\
& $\mathrm{~m}+\mathrm{n}=23550 \pm 10$ & $\mathrm{~m}+\mathrm{n}=23558 \pm 10$ & $3598 \pm 20$ & 3583 \\
& $\mathrm{~m}+\mathrm{n}=43585 \pm 10$ & & & \\
\hline West cavity & $\mathrm{m}+\mathrm{n}=13570 \pm 40$ & $\mathrm{~m}+\mathrm{n}=13643 \pm 20$ & $3650 \pm 20$ & 3624 \\
& $\mathrm{~m}+\mathrm{n}=23540 \pm 20$ & $\mathrm{~m}+\mathrm{n}=23614 \pm 10$ & & \\
\hline
\end{tabular}

The values of the radius of curvature are deduced from the Fabry-Perot signals and from the mirror map measurement (last 3 columns) for the two Fabry-Perot cavities of Virgo.

where $d_{\mathrm{USB}-\text { carrier }}$ is the cavity length difference between the upper sideband and the carrier $\mathrm{TEM}_{00}$ resonances. The upper sideband resonance is well observed for a well aligned cavity as shown on Figure 10. Since the modulation frequency is known the cavity length can be determined from (15). This gives: $L_{\text {north }}=(2999.86 \pm 0.03) \mathrm{m}$ which is compatible with the expected length $(2999.90 \mathrm{~m})$.

\section{D. Cavity losses}

The Fabry-Perot cavity losses can be due to mirror absorption, scattering (due to rugosity) or long range surface defects which couple the $\mathrm{TEM}_{00}$ mode to higher-order modes. The first kind of losses (absorption and scattering) lead to the loss of some power inside the interferometer while the long range surface defects couple the $\mathrm{TEM}_{00}$ to higher order modes so that the power is not necessarily lost, but both result in a decrease of the Fabry-Perot reflectivity for the $\mathrm{TEM}_{00}$.

The mirror absorption and scattering have been measured after the coating and the total is of the order of $10 \mathrm{ppm}$ for each mirror. A small fraction (typically $50 \mathrm{ppm}$ ) of the beam is transmitted by the end mirror to the end benches. The effect of long range surface defects is estimated with simulations including the measurement of the mirror surface maps.

The losses inside the Fabry-Perot cavity lower their reflectivity and as a consequence lowers the recycling gain, so it is therefore important to keep them as small as possible.

The reflectivity (in amplitude) of the cavities is given by:

$$
r_{F P}=\frac{r_{i}-r_{e}(1-L)}{1-r_{i} r_{e}}
$$

where $r_{i(e)}$ is the reflectivity of the input (end) mirror and $L$ the round trip losses. This reflectivity can in principle be measured with a free cavity by comparing the power 
reflected by the cavity out of resonance to the power reflected on the $\mathrm{TEM}_{00}$ resonance: $r_{F P}^{2}=P_{\text {on res }} / P_{\text {out res }}$. Unfortunately this does not give a precise measurement due to dynamical effects and power fluctuations with the alignment. With this method the power reflectivity was estimated to be in the range 96 to $98 \%$ for both cavities.

It is nevertheless possible to estimate the fraction of the input power which is lost due to scattering inside the Fabry-Perot cavities: from the power measured by the photodiodes at the interferometer pickups (the end mirrors transmitted beams and the interferometer transmitted and reflected beams) it can be deduced that about $35 \%$ of the power incident on the interferometer is lost inside the interferometer. The simulation also shows that $33 \%$ of the input power is lost in the Fabry-Perot cavities due to the mirror surface defects. This power loss is equivalent to round trip losses of $330 \mathrm{ppm}$ inside each arm. It can be concluded that the losses due to long range surface defects which scatter the light at relatively small angle are well reproduced by the simulation.

\section{Recycling cavity}

The lengths of the recycling cavity are of particular importance since the asymmetry between the two arms defines the transmission of the sideband signals to the dark fringe and the mean recycling length should fulfill the resonance condition for the sidebands. The other important quantity is the recycling gain which directly affects the shot noise limit of the sensitivity.

\section{A. Lengths}

\section{A.1. Michelson length asymmetry}

The use of the modulation technique requires that the small Michelson is asymmetric. This asymmetry is defined by $\Delta l=l_{2}-l_{1}$. The sidebands transmission depends on this asymmetry. Therefore this quantity is also needed in order to estimate the expected sidebands transmission (see Section 8.C).

The beam reflected by the West cavity travels $2 \Delta l$ more than the beam reflected by the North arm. Therefore the optimum demodulation phase of this beam should be different by $\Delta \phi=2 \frac{\Delta l \Omega}{c}$. The measurement makes use of this property: the two cavities are alternatively locked and the demodulation phase tuned with a precision of 0.1 degree. The asymmetry deduced from this measurement is: $\Delta l=(0.844 \pm 0.013) \mathrm{m}$.

\section{A.2. Recycling cavity length}

In order to reach the best sensitivity both the carrier and the sidebands should resonate inside the recycling cavity. The condition of resonances are:

$$
\frac{\omega}{c} 2 l_{\text {rec }}=0[2 \pi]
$$


for the carrier and

$$
\frac{\omega \pm \Omega}{c} 2 l_{\text {rec }}=\pi+\phi_{F P}[2 \pi]
$$

for the sidebands, where $\phi_{F P}$ is the phase introduced by the Fabry-Perot cavities on the sidebands. Once the microscopic condition (17) is fulfilled, (18) transforms into a macroscopic condition on the recycling length:

$$
\frac{\Omega}{c} 2 l_{r e c}=\pi+\phi_{F P}[2 \pi]
$$

An analytical calculation and a simulation study taking into account the dephasing due to the Fabry-Perot cavities shows that the optimum value is $l_{\text {rec }}=12.073 \mathrm{~m}$. The recycling length should fulfill this condition within $\pm 1 \mathrm{~cm}$. It was also found with the simulation including the mirror maps (see Section 3) that these have a non negligible impact on the optimal recycling length and it could be several centimeters different from this last value. But since the beam splitter transmission map had not been measured at 45 degrees no conclusion could be drawn.

Several independant measurements of this length have been done:

- it has been deduced from the measurement of the position of the mirror suspension point during the installation and the known position of the reflective surface of the mirrors with respect to this point: $l_{\text {rec }}=(12.07 \pm 0.01) \mathrm{m}$.

- the optical length of the recycling cavity has been measured during the commissioning of the central part of Virgo (the $\mathrm{CITF}^{23}$ ) using an auxiliary laser: it is deduced from the measurement of the free spectral range of this cavity obtained from transfer function between frequency noise and the interferometer output signal. This measurement cannot be performed on Virgo since the laser beam is now filtered by the input mode cleaner cavity which makes the measurement less straightforward. Taking into account the changes of position of the mirrors for Virgo with respect to the CITF gives: $l_{\text {rec }}=(12.07 \pm 0.01) \mathrm{m}$.

- the last measurement is done with the Virgo final configuration and relies on the fact that if the sidebands are at resonance the field comes back in phase with the incoming field, otherwise some phase shift is accumulated proportionnally to the mean number of round trips i.e. to the recycling gain. In this last case the demodulation phase should shift from the case without recycling. The interferometer has been locked in the recombined and in the recycled configuration and the demodulation phase of the dark fringe has been adjusted. A phase difference lower than 2 degrees has been measured, this indicates that the recycling cavity length is the correct one within about $1 \mathrm{~cm}$.

These three measurements agree within each other. The third one is the more direct and confirms that the recycling length is within $1 \mathrm{~cm}$ of the optimal value. 


\section{B. Recycling gains}

The recycling gains depend on the power recycling mirror reflectivity and the Fabry-Perot reflectivity for the carrier

$$
G=\left(\frac{t_{P R}}{1+r_{P R} r_{F P}}\right)^{2}
$$

as well as the small Michelson asymmetry for the sidebands:

$$
G_{S B}=\left(\frac{t_{P R}}{1-\cos \left(\frac{\Omega \Delta l}{c}\right) r_{P R} r_{F P}^{S B}}\right)^{2}
$$

where $t_{P R}$ and $r_{P R}$ are the PR amplitude transmission and reflectivities, $r_{F P}$ (resp. $r_{F P}^{S B}$ ) the average Fabry-Perot reflectivity for the carrier (resp. sidebands), $\Omega$ the modulation frequency and $\Delta l$ the small Michelson asymmetry. Without losses in the Fabry-Perot cavities, when these are resonant on the carrier, $r_{F P}=-1$ and $r_{F P}^{S B}=+1$ and given a PR mirror reflectivity equal to $92.2 \%$ the recycling gains are expected to be $G=50$ and $G_{S B}=36$.

These gains can be deduced from the ratio of the power incident on the beam splitter mirror with and without recycling:

$$
G^{00}=T_{P R} \frac{P_{D C}^{\text {recycled }}}{P_{D C}^{\text {recombined }}} \quad G_{S B}^{00}=2 T_{P R} \frac{P_{2 \Omega}^{\text {recycled }}}{P_{2 \Omega}^{\text {recombined }}}
$$

where $P_{D C(2 \Omega)}^{\text {recombined(recycled) }}$ is the DC power (resp. the power at twice the modulation frequency) in the recombined (resp. recycled) configuration. The recycling gains extracted from these measurements are: $G=31 \pm 1$ and $G_{S B}=19 \pm 1$. Taking into account the mismatching of the input beam with the Fabry-Perot cavities (see Section 6.A) the deduced recycling gains for a well matched $\mathrm{TEM}_{00}$ should be:

$$
G^{00}=33 \pm 1 \quad G_{S B}^{00}=20 \pm 1
$$

These gains are significantly smaller than those computed above from scalar values. This is expected if there are non negligible losses in the Fabry-Perot cavities. A simulation (see Section 3) including the mirror long range surface defects which have been measured after coating gives also smaller recycling gains: $G=34$ and $G_{S B}=30$. It is clear from this simulation that these low gain values are due to the surface defects of the mirrors. The carrier is mainly sensitive to defects inside the Fabry-Perot cavities (see 7.D) while the sidebands are mainly sensitive to the recycling cavity defects since they do not couple to the Fabry-Perot cavities. The gain of the carrier obtained in simulation agrees well with the measurement while the gain of the sidebands is significantly higher in simulation. This

might be due to the fact that the beam splitter mirror is not well simulated: the surface map 
was only measured at zero degrees and not at 45 degrees. Moreover, as shown in Figure 11 the gain of the sidebands is also sensitive to the residual radius of curvature which is not known for the beam splitter mirror (the residual radius of curvature of the power recycling mirror is around $30 \mathrm{~km}$ ). The gain of the sidebands is also sensitive to the length of the recycling cavity: simulation shows that it varies by \pm 1 for a variation of $\pm 1 \mathrm{~cm}$.

Assuming that all the losses are located inside the Fabry-Perot cavities, the mean FabryPerot reflectivity for the carrier, deduced from (20) is therefore: $R_{F P}=0.982 \pm 0.002$. This is equivalent to mean round trip losses of $(570 \pm 50) \mathrm{ppm}$ (including the end mirror transmission which is around $50 \mathrm{ppm}$ ). This gives only an upper limit of the Fabry-Perot losses since the recycling gain might also be lowered by defects inside the recycling cavity, as it is observed in simulation.

Assuming $R_{F P}=r_{F P}^{2}=0.982$ the maximum recycling gain which could be achieved taking the same reflectivity for the PR mirror would be 55. A new PR mirror has being installed with a reflectivity of $95 \%$ which allowed, as expected, the increase of the carrier recycling gain up to 43 .

\section{C. Transmission of the sidebands}

For a non perfect contrast the higher the transmission of the sidebands the smaller the shot noise limit (see Equation 1) therefore the sidebands transmission has to be maximized. For the recycled interferometer it is given by:

$$
T=G_{S B} \sin ^{2}\left(\frac{\Omega \Delta l}{c}\right)=\left(\frac{t_{P R} r_{F P}^{S B} \sin \left(\frac{\Omega \Delta l}{c}\right)}{1-\cos \left(\frac{\Omega \Delta l}{c}\right) r_{P R} r_{F P}^{S B}}\right)^{2}
$$

From the Virgo design, neglecting losses, one expects $T=0.45$. Since this quantity is proportional to the recycling gain, the measurement of the recycling gain (presented in Section 8.B) suggests a lower value: $T=0.25$.

The dark fringe signal is also demodulated at twice the modulation frequency allowing a measurement of the sidebands power: $P_{2 \Omega}=2 T_{\text {opt }} P_{0} T J_{1}(m)^{2}$ where $T_{\text {opt }}$ is the transmission of the dark fringe from the interferometer to the photodiode. Unfortunately there are significant uncertainties on quantities entering this equation (about $10 \%$ on $P_{0}$ and $J_{1}$ ) therefore the sidebands transmission is not precisely measured: $T=0.12 \pm 0.03$. It is significantly smaller than what is expected from the sidebands recycling gain. This might indicate that the sidebands contain a large fraction of high-order modes since $T$ is measured after the output mode cleaner while $G_{S B}$ is not. 


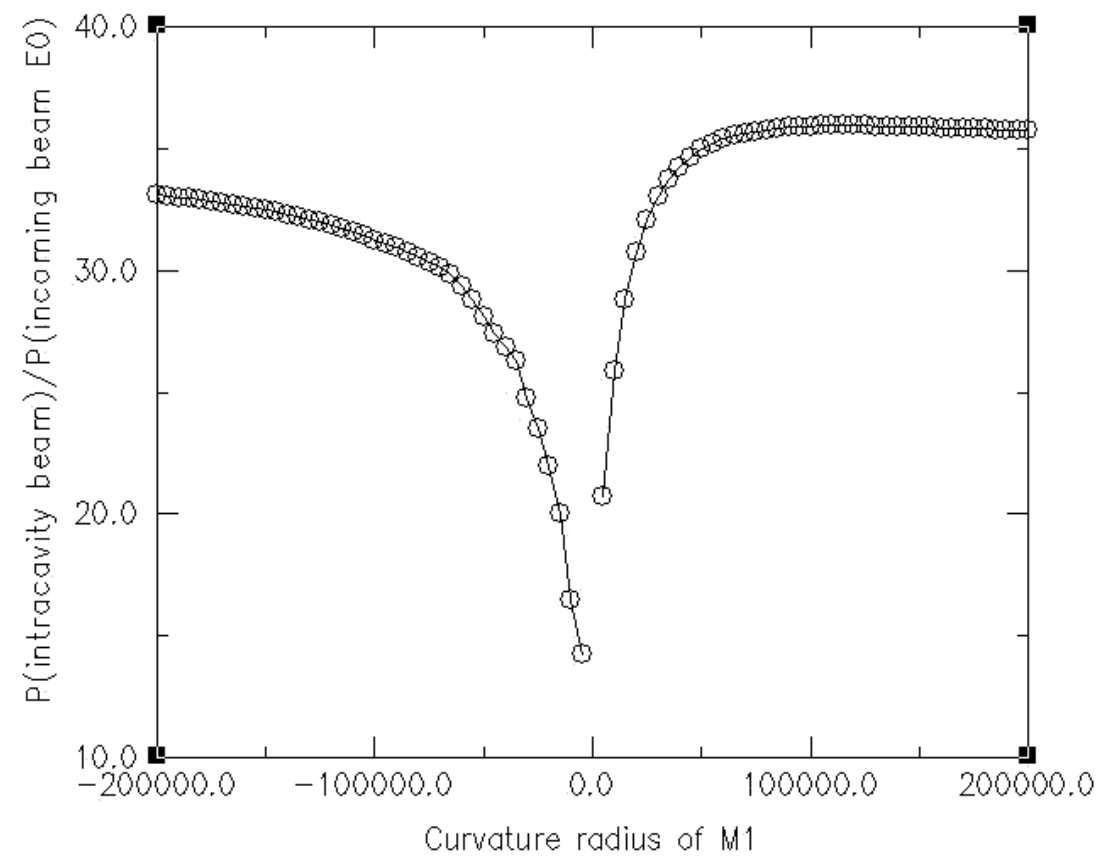

Fig. 11. Recycling gain of the sidebands as predicted by simulation as a function of the radius of curvature (in meters) of the power recycling mirror. The simulation is done for a simple cavity, the mirror transmissions are set respectively to $7.8 \%$ and $1.4 \%$ so that the expected gain corresponds to the maximum gain of the sidebands in Virgo $(G=36)$. 


\section{Contrast defect}

The contrast defect is defined by twice the ratio of the carrier transmitted power to the power incident on the beam splitter mirror. Since the interferometer is locked on the dark fringe it should be null for a perfect interferometer. However there is always a small contrast defect due to the mirror defects, mode mismatch to the cavities and the alignment imperfections (due to finite gain of the alignment loops): these create higher order modes which are not on the dark fringe.

Taking into account only the asymmetry of the Fabry-Perot field reflectivities which, from simulations is expected to be of the order of $0.2 \%$, the resulting contrast defect is of the order of few $10^{-6}$. A full simulation including higher order modes shows that a typical contrast defect is of the order of $10^{-2}$. As can be seen on Figure 2 the shot noise limited sensitivity improves significantly if the contrast defect is reduced below $10^{-4}$.

The contrast is improved in Virgo by means of an output mode cleaner ${ }^{14,15}$ which rejects higher order modes. In the following the contrast before and after the output mode cleaner (OMC) are given for two optical configurations: the so-called recombined and recycled configurations. In the recombined configuration the recycling technique is not used: the power recycling mirror is misaligned, the Fabry-Perot cavities are kept at resonance and the Michelson on the dark fringe. The recycled configuration refers to the full Virgo, i.e. including the power recycling technique.

\section{A. Recombined interferometer}

For the recombined interferometer the total DC power on the dark fringe is given by

$$
P_{D C}=P_{0}\left(J_{0}(m)^{2} \frac{1-C}{2}+2 T_{\text {recomb }} J_{1}(m)^{2}\right)
$$

where, in this case $T_{\text {recomb }}$, the transmission of the sidebands is here given by $T_{\text {recomb }}=$ $\sin ^{2}\left(\frac{\Omega \Delta l}{c}\right)$. The second term is the power of the sidebands and is estimated from the expected value of $T_{\text {recomb }}$. This term is negligible compared to the power on the dark fringe before the output mode cleaner and the contrast defect is easily deduced from the measurement of the DC power: $1-C=3 \times 10^{-4}$ before the output mode cleaner. After the output mode cleaner the total power is comparable to the expected contribution of the sidebands, therefore only an upper limit has been put: $1-C<10^{-4}$.

\section{B. Recycled interferometer}

When the interferometer is in the recycled configuration the total DC power on the dark fringe is the sum of a contribution of the carrier and of the sidebands:

$$
P_{D C}=P_{0}\left(G J_{0}(m)^{2} \frac{1-C}{2}+2 T J_{1}(m)^{2}\right)
$$


The second term is identical to the power demodulated at twice the modulation frequency (see 8.C) and can be subtracted to determine the contrast defect $(1-C)$. The mean power before the $\mathrm{OMC}$ is $6.6 \mathrm{~mW}$ whereas after it is reduced to $1.6 \mathrm{~mW}$ out of which $(1.2 \pm 0.2) \mathrm{mW}$ is the sidebands contribution. Therefore the contrast defect is: $1-C=3 \times 10^{-4}$ before the $\mathrm{OMC}$ and $1-C=3 \times 10^{-5}$ after. The observed contrast defect before the output mode cleaner is quite good, while it was predicted to be $1 \%$ by the simulation. This difference is not yet understood.

Nevertheless, as illustrated in Figure 2, using the OMC allows to improve the sensitivity by $35 \%$ for a modulation index $m \approx 0.15$. Without an output mode cleaner the modulation depth would have to be increased up to its maximum tunable value (around 0.4) in order not to reduce the sensitivity by more than $10 \%$.

\section{Optical gain for the gravitational wave signal}

The optical gain which converts the interferometer differential arm length variation (in meters) into the output port photodiode signal (in Watts) is given by:

$$
O G=4 P_{i n}^{00} T_{d f} J_{0}(m) J_{1}(m) \frac{2 F}{\pi} \frac{2 \pi}{\lambda} \sqrt{G T} \mathrm{~W} / \mathrm{m}
$$

where $P_{i n}^{00}$, the incident power coupled to the $\mathrm{TEM}_{00}$ mode, is given by the product of the input power $P_{i n}$ and of the beam matching to the cavities $M$. During the first period of the recycled interferometer commissioning which is studied in this article (until end 2005), the power exiting the input mode cleaner was reduced by a factor 10 before entering the interferometer. The input power was deduced from the output port power measurements and from the input mode cleaner transmittance (see section 5): $P_{i n}=(0.9 \pm 0.1) \mathrm{W} . T_{d f}$ is the transmission from the interferometer output port to the photodiodes measuring the dark fringe signal: $T_{d f}=0.85 \pm 0.05$. It is mainly given by the transmission of the output mode cleaner (90\%) and that of the Faraday isolator (96\%) located between the output mode cleaner and the photodiodes. The measurement of the other parameters entering in the expression (27) are reported in this paper: $M=0.94, m=0.16 \pm 0.01, G=33 \pm 1, T=0.12 \pm 0.03$ and $F=50 \pm 1$. Therefore the expected optical gain is $O G=(0.8 \pm 0.2) \times 10^{8} \mathrm{~W} / \mathrm{m}$.

The optical gain has been measured with the calibration procedure. To this purpose permanent lines (at frequencies around $350 \mathrm{~Hz}$ ) are added to the correction sent to the cavities end mirror. The amplitude of these lines is converted into meters using the actuator electronic and mechanical transfer function. The optical gain is then the ratio of the amplitude of the signal measured on the dark fringe at this frequency to the amplitude of the mirror displacement. The optical gain measured with this procedure was $O G=(0.71 \pm 0.15) \times 10^{8} \mathrm{~W} / \mathrm{m}$ where the uncertainty reflects the uncertainty on the mirror actuator transfer function. Therefore the measured optical gain agrees with the expected optical gain. 
At the end of 2005, before the commissioning run called $\mathrm{C} 7$, the modulation index was increased by roughly a factor 2. Its value was measured with the same method as described in Section 4.A: $m=0.30 \pm 0.01$. In this case the expected optical gain is $O G=(1.5 \pm 0.3) \times 10^{8} \mathrm{~W} / \mathrm{m}$. This value is compatible with the measured optical gain $O G=(1.0 \pm 0.2) \times 10^{8} \mathrm{~W} / \mathrm{m}$.

\section{Conclusion}

The techniques developed for the measurement of the Virgo optical parameters have been presented in this paper. The good agreement observed between these measurements and the predictions made with the simulation using the measured mirror maps allows the understanding of the optical characteristics of the interferometer and gives confidence in the simulation for future upgrade studies.

It has been shown that, except for the recycling gain, the measured optical parameters meet the design specifications reported in. ${ }^{2}$ It can in particular be noticed that the contrast defect of the interferometer is good, and better than expected. Nevertheless, the use of an output mode cleaner leads to an improvement of the sensitivity by about $35 \%$. Concerning the smaller value of the recycling gain, it was possible to understand that this gain was limited by losses inside the Fabry-Perot cavities. Some upgrades can therefore be designed based on these observations. In particular since the power recycling mirror has been changed recently, its reflectivity has been increased in order to obtain a higher recycling gain. This value was fixed on the basis of the measurement of the Fabry-Perot reflectivities. Moreover, it was shown that the main contribution to losses is due to long range surface defects of the mirrors inside the Fabry-Perot cavities. When these mirrors will be changed a corrective coating could be performed in order to obtain a more regular surface and therefore reduce these losses.

Following each modification in the Virgo optical configuration the techniques presented here will be used in order to measure the interferometer optical properties.

\section{References}

1. C. Bradaschia et al., NIM in Physics Research A289, 518-525(1990)

2. The Virgo Collaboration, Final Design Report (1997), Virgo technical note VIR-TREDIR-1000-13.

3. F Acernese et al., The status of Virgo, PoS(HEP2005)029.

4. D. Sigg, Class. Quantum Grav 23 (2006) S51.

5. H. Lueck et al., Class. Quantum Grav. 23 (2006) S71.

6. K. Hayama, M. Fujimoto, Class. Quantum Grav. 23 (2006) S9.

7. M. E. Gersenshtem, V.I. Pustovoit, Sov. Phys. - JETP 16 (1963) 433. 
8. R. Weiss, Quaterly progress report of the research laboratory electronics, MIT 105 (1972) 379.

9. R. W. P. Drever, in: T. Piran, N. Deruelle (Eds), Gravitational Radiation Proc, Les Houches Summer Institute, North-Holland, Amsterdam, 1982, p.321.

10. R. V. Pound, Electronic Frequency Stabilization of Microwave Oscillators, Rev Sci. Instrum. 17 490-505 (1946)

11. R. W. P. Drever et al., Laser phase and frequency stabilization using an optical resonator, Appl. Phys. B:Photophys. Laser Chem. 31 (1983) 97-105

12. T.M. Niebauer et al., Nonstationary shot noise and its effect on the sensitivity of interferometers. Phys. Rev. A 43, 5022 (1991)

13. F. Beauville et al., The VIRGO large mirrors: a challenge for low loss coatings, Class. Quantum Grav. 21 (2004) S935-S945

14. The Virgo Collaboration, Interferometer signal detection system for the VIRGO experiment, Class. Quantum Grav. 19 (2002) 1857-1863.

15. F. Beauville et al., Improvement in the shot noise of a laser interferometer gravitational wave detector by means of an output mode-cleaner, Class. Quantum Grav. 23 (2006) 3235-3250

16. D. Babusci, H. Fang, G. Giordano, G. Matone, L. Matone and V. Sannibale, Alignment procedure for the VIRGO interferometer: experimental results from the Frascati prototype Phys. Lett A 226 (1997) 31.

17. D. Z. Anderson, Alignment of resonant optical cavities, Appl. Opt. 23 2944-2949 (1984)

18. J-Y. Vinet, P. Hello, C.N. Man, A. Brillet, A high accuracy method for the simulation of non-ideal optical cavities, J. Phys. I 2, 1287-1303 (1992).

19. A. Brillet, J-Y. Vinet, V. Loriette, J-M. Mackowski, L. Pinard, A. Remillieux, Virtual gravitational wave interferometers with actual mirrors, Phys. Rev. D 67 (2003) 102006.

20. F. Bondu and O. Debieu, Formulae for Fabry-Perot cavity accurate parameter measurement via optical transfer function, submitted to Applied Optics.

21. B. Caron et al., A time domain, general purpose simulation program for the VIRGO experiment, Astropart. Phys., 10 (1999) 369-386.

22. A. Freise, G. Heinzel, H. Luck, R. Schilling, B. Willke and K. Danzmann, Frequency domain interferometer simulation with higher-order spatial modes Class. Quantum Grav. 21 (2003) 1067-1074.

23. F. Acernese et al., The commissioning of the central interferometer of the Virgo gravitational wave detector, Astropart. Phys. 21 (2004) 1-22 